\title{
Emergence of Viral hemorrhagic septicemia virus in the North American Great Lakes region is associated with low viral genetic diversity
}

\author{
Tarin M. Thompson ${ }^{1}$, William N. Batts ${ }^{1}$, Mohamed Faisal ${ }^{2}$, Paul Bowser ${ }^{3}$, \\ James W. Casey ${ }^{3}$, Kenneth Phillips ${ }^{4}$, Kyle A. Garver ${ }^{5}$, James Winton ${ }^{1}$, Gael Kurath ${ }^{1, *}$ \\ ${ }^{1}$ Western Fisheries Research Center, U.S. Geological Survey, 6505 NE 65th Street, Seattle, Washington 98115, USA \\ ${ }^{2}$ Aquatic Animal Medicine, Department of Pathobiology and Diagnostic Investigation, College of Veterinary Medicine, \\ Michigan State University, S-110 Plant Biology Building, East Lansing, Michigan 48824, USA \\ ${ }^{3}$ Aquatic Animal Health Program, Department of Microbiology and Immunology, College of Veterinary Medicine, \\ Cornell University, Ithaca, New York 14853, USA \\ ${ }^{4}$ La Crosse Fish Health Center, USFWS, 555 Lester Avenue, Onalaska, Wisconsin 54650, USA \\ ${ }^{5}$ Fisheries \& Oceans Canada, Pacific Biological Station, 3190 Hammond Bay Road, Nanaimo, British Columbia V9T 6N7,
} Canada

\begin{abstract}
Viral hemorrhagic septicemia virus (VHSV) is a fish rhabdovirus that causes disease in a broad range of marine and freshwater hosts. The known geographic range includes the Northern Atlantic and Pacific Oceans, and recently it has invaded the Great Lakes region of North America. The goal of this work was to characterize genetic diversity of Great Lakes VHSV isolates at the early stage of this viral emergence by comparing a partial glycoprotein (G) gene sequence (669 nt) of 108 isolates collected from 2003 to 2009 from 31 species and at 37 sites. Phylogenetic analysis showed that all isolates fell into sub-lineage IVb within the major VHSV genetic group IV. Among these 108 isolates, genetic diversity was low, with a maximum of $1.05 \%$ within the 669 nt region. There were 11 unique sequences, designated vcG001 to vcG011. Two dominant sequence types, vcG001 and vcG002, accounted for $90 \%$ (97 of 108) of the isolates. The vcG001 isolates were most widespread. We saw no apparent association of sequence type with host or year of isolation, but we did note a spatial pattern, in which vcG002 isolates were more prevalent in the easternmost sub-regions, including inland New York state and the St. Lawrence Seaway. Different sequence types were found among isolates from single disease outbreaks, and mixtures of types were evident within 2 isolates from individual fish. Overall, the genetic diversity of VHSV in the Great Lakes region was found to be extremely low, consistent with an introduction of a new virus into a geographic region with previously naïve host populations.
\end{abstract}

KEY WORDS: Emerging infectious diseases • Fish disease • Fish rhabdovirus · Genotyping • Molecular epidemiology • Phylogenetic analysis . Viral haemorrhagic septicaemia virus . Viral hemorrhagic septicemia virus · VHSV

\section{INTRODUCTION}

Viral hemorrhagic septicemia virus (VHSV) is a fish rhabdovirus that has emerged dramatically in the Great Lakes region of North America since its first iso- lation there in 2003 (Elsayed et al. 2006). The disease, VHS, was first described in Europe in 1938 (Schäperclaus 1938), and the virus itself has historically been considered a European fish pathogen because of its severe impact on trout farms in western Europe since 
the 1950s (Wolf 1988, Smail 2000, Skall et al. 2005). Because outbreaks of VHSV are associated with acute systemic disease, hemorrhage, and mortality rates that can mount up to $90 \%$ in a broad range of susceptible species, VHSV is listed by the World Organization for Animal Health (formerly the Office International des Epizooties, still referred to as OIE) as one of only 9 reportable fish diseases (OIE 2010). In 1988, VHSV was first detected on the Pacific coast of North America (Meyers \& Winton 1995). Subsequent surveys revealed an extensive reservoir of VHSV in wild marine fish of the northeast Pacific Ocean, and VHS epidemics have been reported in wild Pacific herring and sardines (Meyers \& Winton 1995, Hedrick et al. 2003). VHSV has also been isolated from wild and farmed olive flounder Paralichthys olivaceus in Japan and Korea (Nishizawa et al. 2002, Kim et al. 2003). Subsequent surveys in European waters also found an extensive VHSV reservoir involving many marine fish species (Skall et al. 2005). Therefore, prior to its recent emergence in the Great Lakes, VHSV was considered to be a marine fish virus that had adapted in the mid 1900s to cultured freshwater rainbow trout in Europe (EinerJensen et al. 2004).

The emergence of VHSV in the Great Lakes represents a geographic invasion of the world's largest freshwater resource, and it is the first time VHSV has caused large-scale fish kills in wild freshwater fish populations. Between 2005 and 2008 VHSV caused several large outbreaks in the Great Lakes watershed involving freshwater drum Aplodinotus grunniens, muskellunge Esox masquinongy, round goby Neogobius melanostomus, yellow perch Perca flavescens, gizzard shad Dorosoma cepedianum, black crappie Pomoxis nigromaulatus, and bluegill Lepomis macrochirus (Elsayed et al. 2006, Lumsden et al. 2007, Groocock et al. 2007, Kane-Sutton et al. 2010). Currently, 28 species of freshwater fish, covering a wide diversity of taxonomic families, are regulated by the Viral Hemorrhagic Septicemia (VHS) Interim Rule imposed by the US Department of Agriculture-Animal and Plant Health Inspection Service (USDA-APHIS) in response to the emergence of VHSV in the Great Lakes region (USDA-APHIS 2008a,b).

These host species include several important recreational and aquaculture species, but to date the virus has been found only in wild populations, not in farmed fish. Within the Great Lakes watershed the virus has been isolated from fish in Lake St. Clair, Lake Ontario, Lake Huron, Lake Erie, Lake Michigan, the Saint Lawrence Seaway, and most recently Lake Superior. VHSV has also been found in smaller inland lakes in New York, Wisconsin, and Michigan. Beyond the Great Lakes watershed, but within a state that borders Lake Erie, VHSV has been isolated for the first time in fish from an Ohio reservoir within the Mississippi River watershed. In addition to virus isolations, surveillance using a diagnostic quantitative real-time polymerase chain reaction (qRTPCR) assay has shown that, even in the absence of recent mortality episodes, VHSV is widely distributed in Lakes Huron, Erie, and Ontario (Bain et al. 2010).

Prior to the emergence of VHSV in the Great Lakes, phylogenetic studies reported by European researchers defined 4 major genetic groups of VHSV, designated I through IV (Einer-Jensen et al. 2004, Snow et al. 2004). VHSV genogroups I, II, and III are predominantly found in marine fish in waters around Europe. Within genogroup I, most VHSV isolates (from European freshwater trout farms) fall into a sub-lineage called Ia. Genogroup IV contains a small number of virus isolates from the Pacific coast of North America. Recent phylogenies using full-length or partial glycoprotein gene sequences of 2 Great Lakes VHSV isolates have shown that they are most closely related to the previously defined genogroup IV, but that they are sufficiently distinct to suggest a different sub-lineage (Elsayed et al. 2006, Lumsden et al. 2007, Ammayappan \& Vakharia 2009). Therefore, the subgroup containing VHSV isolates from the North Pacific Ocean has been designated sub-lineage IVa, with Great Lakes VHSV isolates being designated sub-lineage IVb (Elsayed et al. 2006). Additionally, phylogenetic analysis of $4 \mathrm{VHSV}$ isolates from wild fish in brackish waters of New Brunswick and Nova Scotia, in eastern Canada, have revealed that they fall within genogroup IV and share the greatest sequence homology with Great Lakes VHSV (Gagné et al. 2007).

Since the initial detection of VHSV in the Great Lakes, numerous state, federal, and academic fish health researchers have made a significant effort to investigate fish kills and to survey for the virus in Great Lakes fish. This has resulted in over 100 isolations of VHSV from various fish species at a wide range of locations and at different times (see Table 2). In the present study we describe genetic typing of the majority of these virus isolates, collected through the end of 2009. The goal of this work was to characterize the genetic diversity of VHSV in the Great Lakes in the early stages of this large-scale emergence event, and to interpret these data for insights into the epidemiology and evolution of the virus. This work does not address actual viral incidence, in that we do not attempt to consider all the viral testing that has been negative for VHSV, and the sampling coverage is not necessarily uniform regarding the number of isolates obtained from any positive sampling event. Rather, we used all available virus isolates, each representing virus from an independent fish or pool of fish, and in some cases, multiple isolates from the same outbreak or the same sampling date and location. 
The full-length genomic sequence of the Great Lakes VHSV index isolate from Lake St. Clair, isolate MI03, has been previously reported (Ammayappan \& Vakharia 2009). The genetic typing reported here utilized a partial glycoprotein gene sequence of 669 nucleotides (nt), referred to as the 'central G' region. This region was selected because it encompasses known sites of variation that reflect the diversity of VHSV isolates (Einer-Jensen et al. 2004) and can be readily sequenced for a large number of virus isolates. Our study included $108 \mathrm{VHSV}$ isolates from both the United States and Canada, collected from 2003 to 2009.

\section{MATERIALS AND METHODS}

Virus isolates. Viruses were isolated from multiple host species (Table 1) by various collaborators according to the protocols detailed in AFS-FHS (2007) or OIE

Table 1. Viral hemorrhagic septicemia virus. Species from which VHSV has been isolated in the Great Lakes region, USA and Canada

\begin{tabular}{|ll|}
\hline Common name & Scientific name \\
\hline Fish & \\
Black crappie & Pomoxis nigromaculatus \\
Bluegill & Lepomis macrochirus \\
Brown bullhead & Ictalurus nebulosus \\
Burbot & Lota lota \\
Channel catfish & Ictalurus punctatus \\
Chinook salmon & Oncorhynchus tshawytscha \\
Common carp & Cyprinus carpio \\
Emerald shiner & Notropis atherinoides \\
Freshwater drum & Aplodinotus grunniens \\
Gizzard shad & Dorosoma cepedianum \\
Lake herring & Coregonus artedi \\
Lake trout & Salvelinus namaycush \\
Lake whitefish & Coregonus clupeaformis \\
Largemouth bass & Micropterus salmoides \\
Muskellunge & Esox masquinongy \\
Northern pike & Esox lucius \\
Pumpkinseed & Lepomis gibbosus \\
Rainbow trout & Oncorhynchus mykiss \\
Rock bass & Amblopites rupestris \\
Round goby & Neogobius melanostromus \\
Sea lamprey & Petromyzon marinus \\
Shorthead redhorse sucker & Moxostoma macrolepidotum \\
Smallmouth bass & Micropterus dolomieu \\
Trout perch & Percopsis omiscomaycus \\
Walleye & Sander vitreus \\
White bass & Morone chrysops \\
White perch & Morone americana \\
Yellow perch & Perca flavescens \\
& \\
Other & \\
Amphipods & Diporeia spp. \\
Piscicolid leech & Myzobdella lugubris \\
\hline & \\
& \\
\hline
\end{tabular}

(2009), using the epithelioma papulosum cyprini (EPC), fathead minnow (FHM), Chinook salmon embryo (CHSE), and/or bluegill fry (BF-2) cell lines. Isolated viruses were sent to the Western Fisheries Research Center, Seattle, WA, as supernatants collected from infected cells, and were maintained at $-80^{\circ} \mathrm{C}$.

Viral RNA extraction and sequence analysis. Extraction of viral RNA and reverse-transcriptase (RT)PCR of the central region of the VHSV glycoprotein was performed according to Elsayed et al. (2006). Briefly, total RNA was extracted from $500 \mu \mathrm{l}$ of viral stock supernatant using TriReagent (Sigma) according to manufacturer's instructions, with transfer RNA (Sigma) added to aid in RNA precipitation. Reverse transcription and amplification of a 914 base pair (bp) region of the viral $\mathrm{G}$ gene was done in 30 cycles of PCR using the central G primers 5'-ACT ACC TAC ACA GAG TGA C-3' and 5'-CAA TTT GTC CCC GAA TAT CAT-3'. Four internal primers 5'-TCC CGT CAA GAG GCC AC-3', 5'-ACA ATG GCA TGC ACG GTG ACA3', 5'-TGC CAT TGT GAG CCC CCA-3', and 5-TTC CAG GTG TTG TTT ACC G-3' were used in individual reactions to sequence the central $\mathrm{G}$ region with a fluorescent dye terminator cycle sequencing kit (Applied Biosystems).

Sequence data were edited using Sequencher software version 4.1, and consensus sequences for each isolate were trimmed to $669 \mathrm{nt}$, representing the central G region, corresponding to nt 3409 to 4077 of the full-length genomic sequence of Great Lakes index strain MI03 (Ammayappan \& Vakharia 2009, GenBank accession no. GQ385941). This region comprises nt 462 to 1130 of the full-length 1609 nt G gene of VHSV (Elsayed et al. 2006, GenBank accession no. DQ401193). Consensus sequences were aligned and compared using MacVector version 7.2.3. Isolates with identical central G sequences were grouped together into 'sequence types', and the specific sequence was given a 'universal sequence designator' (USD) of the format vcG\#\#\#, where 'vcG' indicates the sequence region, VHSV central G, and the following 3 digits are an arbitrary numerical identifier for each sequence. Thus, the USD for the index isolate MI03 is vcG001, and other sequences identified within the data set were designated vcG002 to vcG011. Sequences differing from the predominant sequence types vcG001 and vcG002 (see 'Results') were confirmed in independent repeated sequencing reactions starting with viral RNA. For 2 isolates (TAVgr07-03 and TAVgr07-14) that showed sequence heterogeneity in the consensus sequence by the presence of 2 overlapping peaks at the same nt position in the chromatograms, the heterogeneity was confirmed in both forward and reverse sequencing reactions, and in independent repeated sequencing 
reactions starting with viral RNA. Proportions of the $2 \mathrm{nt}$ at the heterogeneous site were estimated visually from all chromatograms. Central $G$ sequences for a representative virus isolate from 10 of the 11 sequence types were submitted to GenBank as nos. HQ623434 to HQ623443. The central G sequence for type vcG002 had been previously submitted to GenBank as no. EF564588 (Groocock et al. 2007)

Phylogenetic analysis. Phylogenetic trees were generated using a data set that included the 11 sequence types found among all isolates characterized from the Great Lakes, and corresponding central G sequences of VHSV isolates from the North American Pacific coast (Isolate BC99-292, GenBank accession no. DQ401188, Elsayed et al. 2006; AK-90, DQ473302, Hedrick et al. 2003; Makah, U28747, Benmansour et al. 1997); from Japan (JP99Obama25, DQ401191, Elsayed et al. 2006); from the North American Atlantic coast (Isolate CA-NB00-02, Genbank accession no. HQ168405; CANB02-01， EF079897; CA-NB04-01b, EF079898; CANS04-01, EF079899; Gagné et al. 2007); and representatives of European VHSV genotypes Ia, Ib, Ic, Id, Ie, II, and III (Isolate DK-3592B, Genbank accession no. X66134; DK-Mrhabdo, Z93414; DK-2835,
AY546585; FI-ka66, AY546614; GE-1.2, AY546619; DK-1p52, AY546576; DK-1p53, AY546577; DK-4p101, AY546581; DK-4p168, AY546582). Multiple sequence alignments were performed using MEGA version 4 software (Tamura et al. 2007). Phylogenetic analysis was performed using MrBayes 3.1 methods (Ronquist \& Huelsenbeck 2003), using the MCMC approach for specific parameters (Hall 2008). The analysis was run for 1100000 generations, and was sampled every 100 cycles. The substitution model used was generaltime-reversible (GTR). The phylogenetic analysis reached convergence with a split frequencies number under 0.01. Tree drawing was performed using MEGA software.

\section{RESULTS}

\section{Sample set}

The 108 VHSV isolates characterized were collected from 37 sampling sites in the Great Lakes region of North America (Fig. 1). Their collection dates and host species are detailed in Table 2 . All but 3 of the isolates

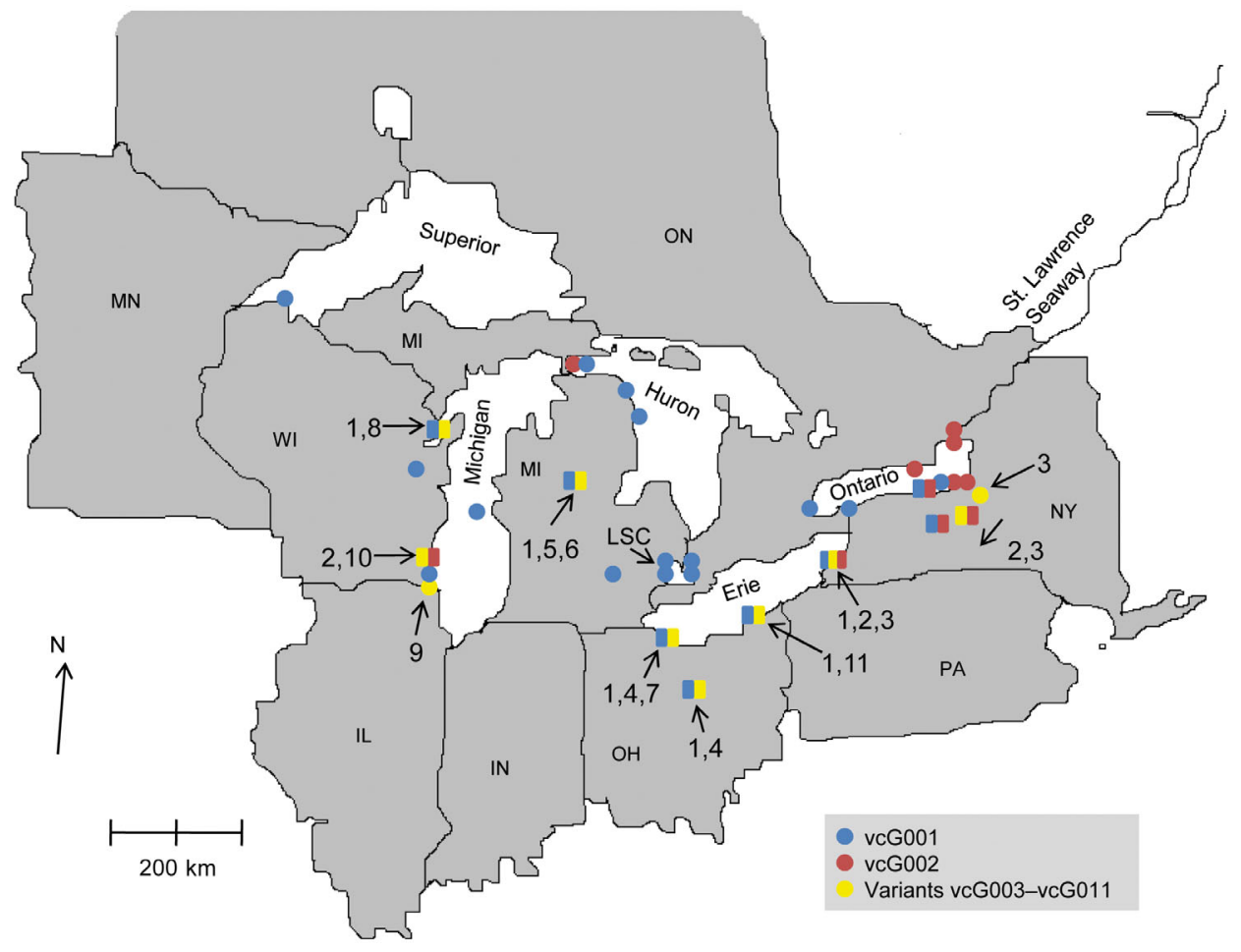

Fig. 1. Viral hemorrhagic septicemia virus. Geographic location of collection sites for VHSV isolates. LSC: Lake St. Clair, MN: Minnesota, WI: Wisconsin, MI: Michigan, IL: Illinois, IN: Indiana, OH: Ohio, PA: Pennsylvania, NY: New York, ON: Ontario. Circles indicate sites where one or more virus isolates had a single sequence type, indicated by color. Rectangles indicate sites where isolates with different genetic types were obtained, indicated by colors within rectangle, with types vcG001 to vcG011 shown as numbers 1 to 11. In many cases multiple isolates were obtained from the same site, on either the same or different dates. Although there are 37 sites, only 33 dots appear because some locations are close enough to one another that they are not separable by symbol 
Table 2. Viral hemorrhagic septicemia virus. Features and genetic sequence types of VHSV isolates from the Great Lakes region used in this study. Additional information on these isolates may be found at http://gis.nacse.org/vhsv. Dates (given as $\mathrm{mm} / \mathrm{dd}$ /yyyy) that include ' $\mathrm{xx}$ ' for the month and/or day indicate unknown sampling dates. Host species scientific binomials are listed in Table 1. Viral sequences are indicated by a universal sequence designator (USD), which identifies a specific 669 nt sequence of the VHSV central glycoprotein (vcG) region

\begin{tabular}{|c|c|c|c|c|}
\hline Location name & Isolate name & Date isolated & Host species & USD \\
\hline \multicolumn{5}{|l|}{ Lake Erie } \\
\hline Dunkirk Harbor & TAVgr07-08 & $04 / 12 / 2007$ & Gizzard shad & vcG002 \\
\hline Dunkirk Harbor & TAVgr07-09a & $04 / 12 / 2007$ & Gizzard shad & vcG001 \\
\hline Dunkirk Harbor & TAVgr07-12 & $04 / 12 / 2007$ & Common carp & vcG003 \\
\hline East Harbor & TAVgr07-20 & $05 / 15 / 2007$ & Bluegill & vcG007 \\
\hline Lake Erie & TAVgr09-05 & $\mathrm{xx} / \mathrm{xx} / 2008$ & Piscicolid leech & vcG001 \\
\hline Middle Basin & TAVgr06-07 & $05 / 31 / 2006$ & Freshwater drum & vcG001 \\
\hline Middle Basin & TAVgr06-08 & $05 / 31 / 2006$ & Walleye & vcG001 \\
\hline Middle Basin & TAVgr06-09 & $05 / 31 / 2006$ & White bass & vcG001 \\
\hline Middle Basin & TAVgr06-10 & $05 / 31 / 2006$ & Yellow perch & vcG001 \\
\hline Middle Basin & TAVgr06-11 & $05 / 31 / 2006$ & Smallmouth bass & vcG001 \\
\hline Middle Basin & TAVgr06-15 & $05 / 31 / 2006$ & Gizzard shad & vcG001 \\
\hline Middle Basin & TAVgr06-53 & $05 / 31 / 2006$ & Trout perch & vcG011 \\
\hline Middle Basin & TAVgr07-21 & $05 / 23 / 2007$ & Yellow perch & vcG001 \\
\hline Middle Basin & TAVgr07-22 & $06 / 07 / 2007$ & Yellow perch & vcG001 \\
\hline Middle Basin & TAVgr08-09 & $05 / 07 / 2008$ & Emerald shiner & vcG001 \\
\hline Middle Basin & TAVgr08-10 & $05 / 20 / 2008$ & Freshwater drum & vcG001 \\
\hline Middle Basin & TAVgr08-11 & $06 / 04 / 2008$ & Freshwater drum & vcG001 \\
\hline Sandusky Bay & TAVgr06-05 & $04 / 26 / 2006$ & Freshwater drum & vcG001 \\
\hline Sandusky Bay & TAVgr06-06 & $04 / 26 / 2006$ & Freshwater drum & vcG001 \\
\hline Western Basin & TAVgr06-12 & $06 / 01 / 2006$ & Yellow perch & vcG001 \\
\hline Western Basin & TAVgr06-13 & $06 / 01 / 2006$ & Yellow perch & vcG001 \\
\hline Western Basin & TAVgr06-14 & $06 / 01 / 2006$ & White bass & vcG001 \\
\hline Western Basin & TAVgr06-16 & $06 / 01 / 2006$ & White bass & vcG004 \\
\hline Western Basin & TAVgr06-17 & $06 / 01 / 2006$ & Walleye & vcG001 \\
\hline Western Basin & TAVgr06-18 & $06 / 01 / 2006$ & Emerald shiner & vcG001 \\
\hline \multicolumn{5}{|l|}{ Lake Huron } \\
\hline Cheboygan Bay & TAVgr06-46 & 08/17/2006 & Lake whitefish & vcG001 \\
\hline Lake Huron & TAVgr08-08 & $06 / 04 / 2008$ & Sea lamprey & vcG002 \\
\hline Swan River weir Huron & TAVgr06- $44^{\mathrm{a}}$ & $09 / 28 / 2006$ & Chinook salmon & vcG001 \\
\hline Thunder Bay & TAVgr06- $43^{\mathrm{a}}$ & $10 / 06 / 2006$ & Walleye & vcG001 \\
\hline Thunder Bay & TAVgr06- $45^{\mathrm{a}}$ & $10 / 06 / 2006$ & Lake whitefish & vcG001 \\
\hline \multicolumn{5}{|l|}{ Lake Michigan } \\
\hline Lake Michigan (central) & TAVgr09-01 & $\mathrm{xx} / \mathrm{xx} / 2008$ & Amphipod & vcG001 \\
\hline Lake Michigan (central) & TAVgr09-02 & $\mathrm{xx} / \mathrm{xx} / 2008$ & Amphipod & vcG001 \\
\hline Lake Michigan (near Milwaukee) & TAVgr08-02 & $06 / 05 / 2008$ & Yellow perch & vcG002 \\
\hline Lake Michigan (near Milwaukee) & TAVgr09-17 & $06 / 05 / 2008$ & Yellow perch & vcG010 \\
\hline North Point Marina & TAVgr08-03 & $06 / 10 / 2008$ & Rock bass & vcG009 \\
\hline Sturgeon Bay & TAVgr07-24 & $05 / 08 / 2007$ & Smallmouth bass & vcG008 \\
\hline Sturgeon Bay & TAVgr09-12 & 05/18/2009 & Smallmouth bass & vcG001 \\
\hline \multicolumn{5}{|l|}{ Lake Ontario } \\
\hline Bay of Quinte & U 13653-1 & 05/xx/2005 & Freshwater drum & vcG002 \\
\hline Bay of Quinte & U $13653-2$ & $05 / x x / 2005$ & Freshwater drum & vcG002 \\
\hline Fairhaven State Park & TAVgr08-06 & $05 / 08 / 2008$ & Round goby & vcG002 \\
\hline Hamilton Harbour & OMNR 5577 & $05 / 10 / 2007$ & Freshwater drum & vcG001 \\
\hline Hamilton Harbour & OMNR 5583 & $05 / 18 / 2007$ & Freshwater drum & vcG001 \\
\hline Irondequoit Bay & TAVgr06-28 & $05 / 15 / 2006$ & Round goby & vcG001 \\
\hline Lake Ontario (near Rochester) & TAVgr07-06 & $03 / 27 / 2007$ & Gizzard shad & vcG001 \\
\hline Lake Ontario (near Rochester) & TAVgr07-07 & $03 / 27 / 2007$ & Gizzard shad & vcG001 \\
\hline Oswego, NY & TAVgr08-05 & $05 / 07 / 2008$ & Round goby & vcG002 \\
\hline Sodus Bay & TAVgr06-29 & $05 / 15 / 2006$ & Smallmouth bass & vcG001 \\
\hline Tibbetts Creek & TAVgr06-30 & $06 / 08 / 2006$ & Smallmouth bass & vcG002 \\
\hline West of Rochester & TAVgr06-01 & $05 / 15 / 2006$ & Round goby & vcG002 \\
\hline
\end{tabular}


Table 2 (continued)

\begin{tabular}{|c|c|c|c|c|}
\hline Location name & Isolate name & Date isolated & Host species & USD \\
\hline \multicolumn{5}{|l|}{ Lake Superior } \\
\hline Apostle Islands & TAVgr10-01 & $12 / 16 / 2009$ & Lake herring & vcG001 \\
\hline \multicolumn{5}{|l|}{ Lake St. Clair } \\
\hline Lake St. Clair & TAVgr05-01 & $07 / 02 / 2003$ & Muskellunge & vcG001 \\
\hline Lake St. Clair & 0601FD & $05 / x x / 2006$ & Freshwater drum & vcG001 \\
\hline Lake St. Clair & 0602SB & $05 / x x / 2006$ & Smallmouth bass & vcG001 \\
\hline Lake St. Clair & 0603BG & $05 / x x / 2006$ & Bluegill & vcG001 \\
\hline Lake St. Clair & TAVgr06-48 & $03 / 14 / 2006$ & Gizzard shad & vcG001 \\
\hline Lake St. Clair & TAVgr06-51 & $04 / 26 / 2006$ & Yellow perch & vcG001 \\
\hline Lake St. Clair & TAVgr06-52 & 05/xx/2006 & Freshwater drum & vcG001 \\
\hline Lake St. Clair & TAVgr06-50 & $05 / 17 / 2006$ & Shorthead redhorse sucker & vcG001 \\
\hline Lake St. Clair & TAVgr06-47 & $05 / 17 / 2006$ & Northern pike & vcG001 \\
\hline Lake St. Clair & TAVgr06-49a & $05 / 17 / 2006$ & Rock bass & vcG001 \\
\hline Lake St. Clair & TAVgr09-03 & $\mathrm{xx} / \mathrm{xx} / 2008$ & Piscicolid leech & vcG001 \\
\hline Lake St. Clair & TAVgr09-04 & $\mathrm{xx} / \mathrm{xx} / 2008$ & Piscicolid leech & vcG001 \\
\hline Lake St. Clair & TAVgr09-10 & $05 / 18 / 2009$ & Muskellunge & vcG001 \\
\hline Lake St. Clair & TAVgr09-11 & $06 / 04 / 2009$ & Smallmouth bass & vcG001 \\
\hline \multicolumn{5}{|l|}{ Michigan-inland } \\
\hline Baseline Lake & TAVgr09-09 & 05/06/2009 & Brown bullhead & vcG001 \\
\hline Budd Lake & TAVgr07-01 & 05/02/2007 & Bluegill & vcG005 \\
\hline Budd Lake & TAVgr07-02 & 05/02/2007 & Black crappie & vcG001 \\
\hline Budd Lake & TAVgr07-03 & 05/02/2007 & Largemouth bass & vcG001/vcG005 \\
\hline Budd Lake & TAVgr07-04 & $05 / 02 / 2007$ & Pumpkinseed & vcG006 \\
\hline \multicolumn{5}{|l|}{ New York-inland } \\
\hline Cayuga-Seneca Canal & TAVgr07-14 ${ }^{\mathrm{a}}$ & $05 / 23 / 2007$ & Pumpkinseed & vcG001/vcG002 \\
\hline Cayuga-Seneca Canal & TAVgr07-15 & $05 / 23 / 2007$ & Pumpkinseed & vcG001 \\
\hline Cayuga-Seneca Canal & TAVgr07-16 & $05 / 23 / 2007$ & Pumpkinseed & vcG001 \\
\hline Little Salmon River & TAVgr07-17a & $04 / 23 / 2007$ & Rainbow trout & vcG003 \\
\hline Ransomville & TAVgr07-10 & $05 / 18 / 2007$ & Bluegill & vcG001 \\
\hline Ransomville & TAVgr07-11 ${ }^{\mathrm{a}}$ & $05 / 18 / 2007$ & Bluegill & vcG001 \\
\hline Skaneateles Lake & TAVgr07-18 & $05 / 08 / 2007$ & Smallmouth bass & vcG002 \\
\hline Skaneateles Lake & TAVgr07-19a & $05 / 08 / 2007$ & Rock bass & vcG002 \\
\hline Skaneateles Lake & TAVgr07-13 & 06/01/2007 & Lake trout & vcG003 \\
\hline \multicolumn{5}{|l|}{ Ohio-inland } \\
\hline Clear Fork Reservoir & TAVgr08-01 & $04 / 21 / 2008$ & Muskellunge & vcG004 \\
\hline Clear Fork Reservoir & TAVgr09-32 & $04 / 22 / 2009$ & Gizzard shad & vcG001 \\
\hline Clear Fork Reservoir & TAVgr09-33 & $04 / 22 / 2009$ & Black crappie & vcG001 \\
\hline \multicolumn{5}{|l|}{ Ontario-inland } \\
\hline Thames River & OMNR 5579 & $05 / 14 / 2007$ & Largemouth bass & vcG001 \\
\hline \multicolumn{5}{|l|}{ Wisconsin-inland } \\
\hline Lake Winnebago & TAVgr07-05 & $05 / 11 / 2007$ & Freshwater drum & vcG001 \\
\hline Oak Creek/Grant Park & TAVgr09-13 & $05 / 28 / 2008$ & Round goby & vcG001 \\
\hline \multicolumn{5}{|l|}{ St. Lawrence Seaway } \\
\hline St. Lawrence River & TAVgr06-03 ${ }^{\mathrm{a}}$ & 05/04/2006 & Round goby & vcG002 \\
\hline St. Lawrence River & TAVgr06-04 & 05/04/2006 & Round goby & vcG002 \\
\hline St. Lawrence River & TAVgr06-27 $7^{\mathrm{a}}$ & $05 / 12 / 2006$ & Burbot & vcG002 \\
\hline St. Lawrence River & TAVgr06- $02^{\mathrm{a}}$ & $05 / 15 / 2006$ & Muskellunge & vcG002 \\
\hline St. Lawrence River & TAVgr06-31 & $06 / 03 / 2006$ & Rock bass & vcG002 \\
\hline St. Lawrence River & TAVgr06-32 & $06 / 03 / 2006$ & Black crappie & vcG002 \\
\hline St. Lawrence River & TAVgr06-33 & $06 / 03 / 2006$ & Black crappie & vcG002 \\
\hline St. Lawrence River & TAVgr06-34 & $06 / 03 / 2006$ & Smallmouth bass & vcG002 \\
\hline St. Lawrence River & TAVgr06-39 & $06 / 03 / 2006$ & Smallmouth bass & vcG002 \\
\hline St. Lawrence River & TAVgr06-19 & $06 / 04 / 2006$ & Brown bullhead & vcG002 \\
\hline St. Lawrence River & TAVgr06-24 & $06 / 04 / 2006$ & Yellow perch & vcG002 \\
\hline
\end{tabular}


Table 2 (continued)

\begin{tabular}{|lcccc}
\hline Location name & Isolate name & Date isolated & Host species & USD \\
\hline St. Lawrence River & TAVgr06-20 & $06 / 05 / 2006$ & White perch & vcG002 \\
St. Lawrence River & TAVgr06-21 & $06 / 05 / 2006$ & Northern pike & vcG002 \\
St. Lawrence River & TAVgr06-26 & $06 / 08 / 2006$ & Smallmouth bass & vcG002 \\
St. Lawrence River & TAVgr06-25 & $06 / 09 / 2006$ & Yellow perch & vcG002 \\
St. Lawrence River & TAVgr06-35 & $06 / 10 / 2006$ & Channel catfish & vcG002 \\
St. Lawrence River & TAVgr06-36 & $06 / 10 / 2006$ & Channel catfish & vcG002 \\
St. Lawrence River & TAVgr06-37 & $06 / 10 / 2006$ & Largemouth bass & vcG002 \\
St. Lawrence River & TAVgr06-38 & $06 / 10 / 2006$ & Smallmouth bass & vcG002 \\
St. Lawrence River & TAVgr06-40 & $06 / 10 / 2006$ & Smallmouth bass & vcG002 \\
St. Lawrence River & TAVgr06-22 & $06 / 12 / 2006$ & Brown bullhead & vcG002 \\
St. Lawrence River & TAVgr06-23 & $06 / 12 / 2006$ & Bluegill & vcG002 \\
St. Lawrence River & TAVgr08-07 & $05 / 18 / 2008$ & Round goby & vcG002 \\
St. Lawrence River & TAVgr08-04 & $05 / 28 / 2008$ & Round goby & vcG002 \\
asolates associated with mortality or clinical signs, or that were epizootic. Other isolates are either from asymptomatic fish or \\
the condition of the fish is unknown & & & & \\
\hline
\end{tabular}

were from the Great Lakes watershed or the St. Lawrence Seaway that flows out from the Great Lakes to the Atlantic Ocean. From the United States, 100 isolates originated from 11 geographic sub-regions defined as follows: Lake Erie, Lake Huron, Lake Michigan, Lake Ontario, Lake Superior, Lake St. Clair, St. Lawrence Seaway, Wisconsin-inland, Michiganinland, Ohio-inland, and New York-inland. From Canada, 8 isolates were obtained at 4 collection sites within 3 sub-regions, including Lake Ontario, Lake St. Clair, and Ontario-inland. These isolates were collected over a $7 \mathrm{yr}$ period from 2003 to 2009. The isolates were obtained from a total of 30 different host types (Table 2), including 28 finfish species, and 2 invertebrates, amphipods of the genus Diporeia collected from Lake Michigan (Faisal \& Winters 2011), and the piscicolid leech Myzobdella lugubris, a parasite of fish in Lakes St. Clair and Erie (Faisal \& Schulz 2009). With regard to disease associations, 16 of the 108 isolates were noted as epidemic, 3 as clinical, 5 as moribund, 13 as associated with some mortality, 28 as asymptomatic, and 43 undefined.

\section{Sequence types}

The central G sequences of the 108 VHSV isolates fell into one of 11 different sequence types designated vcG001 to vcG011. Two of these sequence types included $90 \%$ (97 of 108) of the isolates. Sequence type vcG001 was found in 61 isolates, including the Great Lakes index strain MI03, which was isolated from a muskellunge in 2003 (Elsayed et al. 2006). Another 36 isolates fell into sequence type vcG002. Three sequence types, vcG003, vcG004, and vcG005, were each found in 2 to 3 isolates, and the other 6 sequence types were found in only 1 isolate.
The sequence chromatograms of most isolates indicated a single clear consensus sequence. However, 2 isolates showed apparent genetic heterogeneity, evident as peaks for 2 different nt at the same position in the chromatograms. Isolate TAVgr07-03 from Budd Lake in Michigan was heterogeneous at position 643, with both A ( 45\%) and G ( 55\%). When position 643 is an $A$, the sequence matches vcG001, and when it is a $\mathrm{G}$ it matches vcG005. Isolate TAVgr07-14 from Lake Erie was heterogeneous at position 1060, with both $\mathrm{C}$ $(\sim 40 \%)$ and $\mathrm{G}(\sim 60 \%)$. When this position is a $\mathrm{C}$, it matches vcG001, and when it is a $\mathrm{G}$ it matches vcG002.

\section{Sequence alignment}

Since the vcG001 sequence was found in the Great Lakes index strain MI03 as well as in a majority of the other virus isolates, it was used as our reference sequence for alignments and comparison with the other sequence types vcG002 to vcG011 (Fig. 2). All sequence types except vcG008 and vcG009 differed from vcG001 by only $1 \mathrm{nt}$. Type vcG008 is $2 \mathrm{nt}$ different from vcG001 at positions 653 and 882, and vcG009 is 5 nt different from vcG001 at positions $640,867,885,1022$, and 1060. Collectively, differences occurred at 14 positions, and these positions appeared distributed as several clusters along the length of the $669 \mathrm{nt}$ central G region (Fig. 2A). At one of these positions the same change was found in 2 different sequence types: at position 1060 both vcG002 and vcG009 varied from vcG001 by a single $\mathrm{C}$ to $\mathrm{G}$ substitution. With regard to the nature of the sequence changes, and assuming vcG001 to be the ancestral sequence, $G$ to A transitions were most common, accounting for 6 out of 16 differences. The remainder of the changes were apparently distributed randomly among 7 of the other 11 possible mutations. 


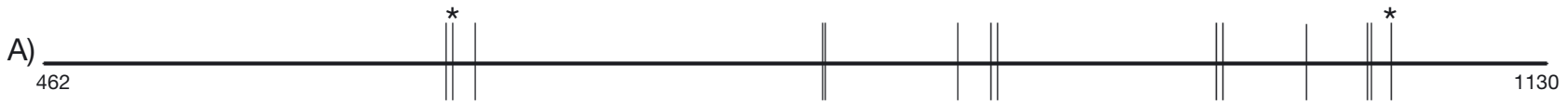

\begin{tabular}{|c|c|c|c|c|c|c|c|c|c|c|c|c|c|c|c|c|}
\hline $\begin{array}{l}\text { VHSV } \\
\text { variant }\end{array}$ & 640 & $643^{*}$ & 653 & 807 & 808 & 867 & 882 & 885 & 982 & 985 & 1022 & 1049 & 1051 & $1060^{*}$ & $\begin{array}{c}\text { Total no. of } n t \\
\text { different from } 001\end{array}$ & $\begin{array}{l}\text { Total no. of } \\
\text { isolates }^{\mathrm{a}}\end{array}$ \\
\hline vcG001 & $A$ & A & $A$ & C & $A$ & $A$ & $\mathrm{G}$ & A & $\mathrm{G}$ & C & $\mathrm{T}$ & $A$ & $T$ & C & -- & 61 \\
\hline 002 & & & & & & & & & & & & & & $G$ & 1 & 36 \\
\hline 003 & & & & & & & & & A & & & & & & 1 & 3 \\
\hline 004 & & & & & & & & & & & & C & & & 1 & 2 \\
\hline 005 & & $\mathrm{G}$ & & & & & & & & & & & & & 1 & 2 \\
\hline 006 & & & & $\mathrm{~A}$ & & & & & & & & & & & 1 & 1 \\
\hline 007 & & & & & & & & & & & & & $\mathrm{~A}$ & & 1 & 1 \\
\hline 008 & & & $\mathrm{G}$ & & & & $\bar{A}$ & & & & & & & & 2 & 1 \\
\hline 009 & $\mathrm{G}$ & & & & & $\mathrm{G}$ & & $\mathrm{G}$ & & & $\mathrm{C}$ & & & $\mathrm{G}$ & 5 & 1 \\
\hline 010 & & & & & $G$ & & & & & & & & & & 1 & 1 \\
\hline 011 & & & & & & & & & & $\mathrm{~T}$ & & & & & 1 & 1 \\
\hline AA changeb & & & $\mathrm{I}_{217} \mathrm{~V}$ & $A_{269 E}$ & & $\mathrm{~K}_{289 \mathrm{R}}$ & $\mathrm{R}_{294 \mathrm{~K}}$ & $\mathrm{~K}_{295} \mathrm{R}$ & & & $\mathrm{S}_{340 \mathrm{P}}$ & $\mathrm{S}_{349 \mathrm{R}}$ & $\mathrm{S}_{350 \mathrm{R}}$ & & & \\
\hline
\end{tabular}

Fig. 2. Viral hemorrhagic septicemia virus. Genetic differences among 11 sequence types identified in Great Lakes VHSV isolates. (A) Horizontal line represents $669 \mathrm{nt}$ central G region sequenced for genetic typing of 108 VHSV isolates from Great Lakes watershed. This region corresponds to nt 3409 to 4077 of the $11184 \mathrm{nt}$ full-length genome sequence of Great Lakes VHSV strain MI03 (GenBank accession no. GQ385941), and to nt 462 to 1130 of the 1609 nt G gene (GenBank accession no. DQ401193). Small vertical lines indicate sites of genetic differences as composite including all sequence variants, and asterisks indicate sites at which genetic heterogeneity was observed within single isolates (see 'Results'). (B) Specific sequence differences in each sequence type (vcG002 to vcG011) are shown relative to vcG001 as a reference sequence. Boxed nt indicate non-synonymous changes that result in amino acid (AA) changes in predicted G protein sequence. ${ }^{\text {a }}$ Total number of isolates shown adds up to 110 due to sequence heterogeneity in 2 isolates. ${ }^{b}$ For sites in which an AA change occurred, first letter shows AA in the vcG001 sequence type. The number represents AA number in reference to G gene. Last letter indicates AA in the variant sequence type

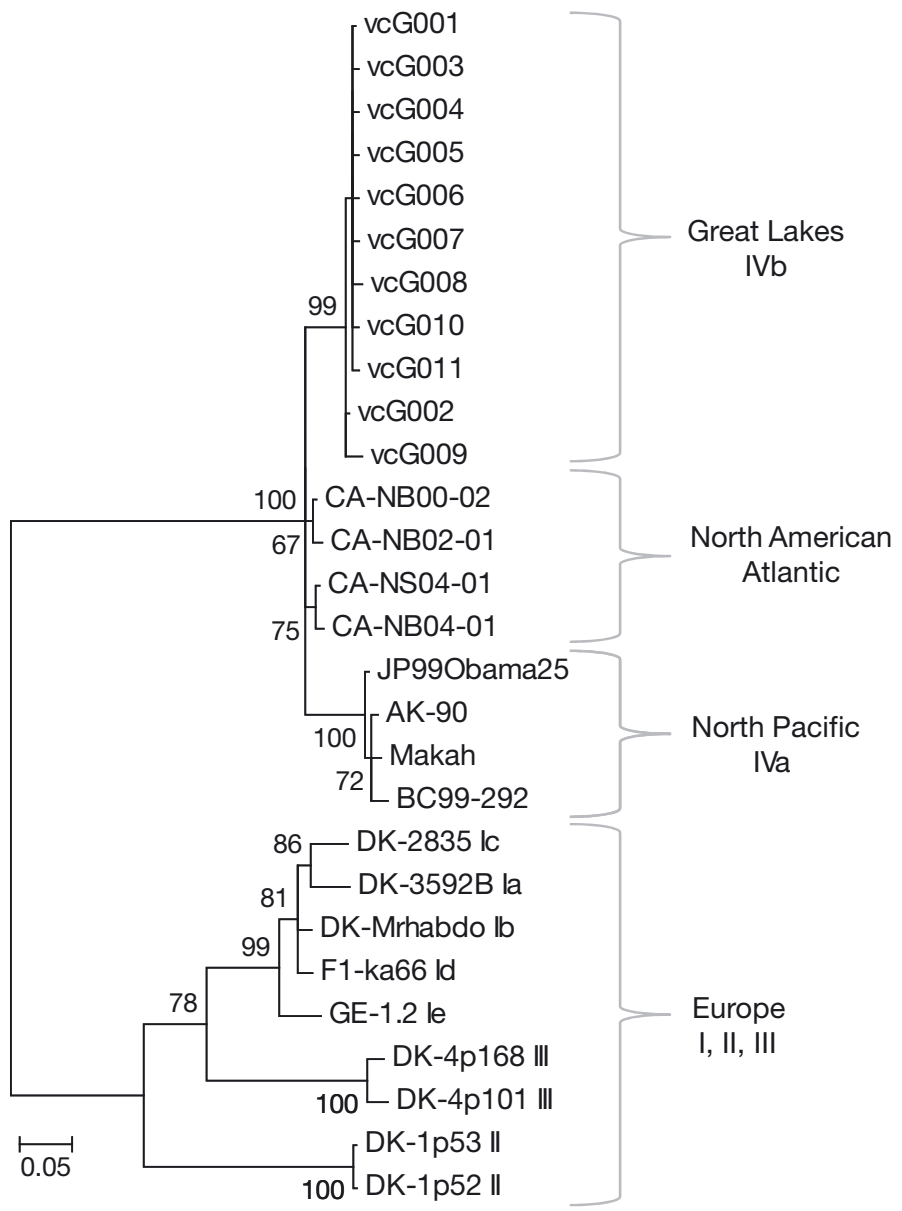

Fig. 3. Viral hemorrhagic septicemia virus. Phylogenetic relationships among Great Lakes VHSV sequence types vcG001 to vcG011, and representative VHSV isolates from Atlantic coast of North America, North Pacific Ocean, and Europe. Phylogenetic analysis used Bayesian methods, with partial glycoprotein (G) nt sequences (669 nt) of 28 different sequences, which encompasses all 4 major VHSV genogroups. Branches for all VHSV from the Great Lakes region are labeled with sequence type (vcG001 to vcG011). The number of virus isolates represented by each sequence type is shown in Fig. 2. Labels for all other branches indicate individual virus isolate designations; for European VHSV isolates, genotype (I, II, or III) and sub-type (a-e) are shown after the isolate name. GenBank accession numbers and references for isolates outside Great Lakes are detailed in 'Materials and methods'. Coalescence values are shown at branch nodes; scale bar indicates the number of substitutions per nt site 
Among the 14 nt differences identified, 8 encoded amino acid changes and 6 were silent. Notably, the single difference between vcG001 and vcG002 was silent; for this reason no amino acid change was identified between these major types for the region examined. Amino acid differences from vcG001 were found in 5 sequence types (vcG004, vcG006, vcG007, vcG008, and vcG009); in the most divergent type, vcG009, 3 amino acid changes were noted among the 5 nucleotide differences.

\section{Phylogenetic analysis}

Phylogenetic analysis using Bayesian methods grouped all Great Lakes VHSV sequences into a single clade of short branches that was strongly supported with a coalescence value of 99 (Fig. 3). This clade contained the sequence type of the previously characterized MI03 isolate, and was thus identified as an expanded sub-lineage IVb. As in previously published trees, the VHSV isolates from the North Pacific Ocean comprised sub-lineage IVa, supported with a coalescence value of 100. The isolates from the Atlantic coastal waters of New Brunswick and Nova Scotia fell on short branches, close to the most recent common ancestral node for sub-lineage IVa and IVb. All genotype IVa and IVb isolates were clearly separate from European VHSV genotypes I, II, and III, which were resolved in a manner consistent with previously published phylogenetic trees (Einer-Jensen et al. 2004, Snow et al. 2004).

\section{Association of sequence types with isolation year or host}

The extreme variation observed in frequency of samples by year, host species, location, and sequence type made statistical inferences problematic, especially considering the non-random sampling design of the data set. Therefore we have examined the data only for general trends, which we present here with the acknowledgement that the associations or lack of associations described are not statistically supported inferences. No association of specific sequence types with year of isolation or with specific hosts was apparent. As shown in Fig. 4A, multiple sequence types were observed within each year during which more than 7 isolates were collected (from 2006 to 2008), with the greatest diversity of variants being found in 2007 . Although the largest number of virus isolates were from 2006, and the number appeared to decline through 2009, this does not necessarily indicate an actual decrease in virus over time, since-due to a large number of fish kills - sampling effort was likely most intense in 2006, and in many cases we received multiple isolates per outbreak. Similar analysis of virus in different hosts found multiple sequence types in each host species from which more than 3 isolates were obtained (Fig. 4B). The distribution of variants appeared to be random among hosts, without grouping according to host families or into predator-prey groups.

\section{Spatial associations}

Spatially, some association was observed between sequence type and geographic location (Fig. 4C). In Lake St. Clair, which is small compared with the 5 Great Lakes (Fig. 1), all 14 isolates obtained between 2003 and 2009 were vcG001, which is the most common type and includes the index isolate MI03. The single isolate from Lake Superior also typed as vcG001. Sequence type vcG001 was also predominant in Lakes Erie (20 of 25 isolates) and Huron (4 of 5 isolates), although other sequence types were also found in these lakes, including vcG002, vcG003, vcG004, vcG007, and vcG011. Lake Michigan exhibited more diversity, with vcG001 in 3 of 7 isolates and 4 sequence types among the other isolates. Lake Ontario isolates were evenly split between vcG001 (6 of 12 isolates), and vcG002 (6 of 12 isolates). Virus isolates from inland waters of New York were vcG001, vcG002, and vcG003. The single virus islate from inland waters of Ontario, Canada was type vcG001. The isolates from inland waters of Wisconsin typed as vcG001. Michigan inland waters revealed types vcG001, vcG005, and vcG006. Similarity, isolates from inland Ohio, in the Mississippi River watershed, had types vcG001 and vcG004.

Presented in a different way, within sequence type vcG001, virus isolates were found in 10 of the 11 designated geographic sub-regions throughout nearly the entire study period. The sole exception was in 2005, when only 2 virus isolates were obtained, and from 22 of the 30 host species represented in the data set. It is therefore clear that vcG001 represents a widely dispersed - and the most common - sequence type.

The most notable spatial trend involved an apparent focus of sequence type vcG002 in the easternmost subregions. All isolates (24 of 24 ) from various hosts in the St. Lawrence Seaway had sequence type vcG002, but 22 of these were collected within a 6 wk period in 2006, and 2 others in 2008. Unlike the other Great Lakes, where vcG001 was predominant, Lake Ontario had equal numbers of isolates with types vcG001 and vcG002, and inland waters of the adjacent New York state had isolates with sequence types vcG001, vcG002, or vcG003. Considering the proximity to one 

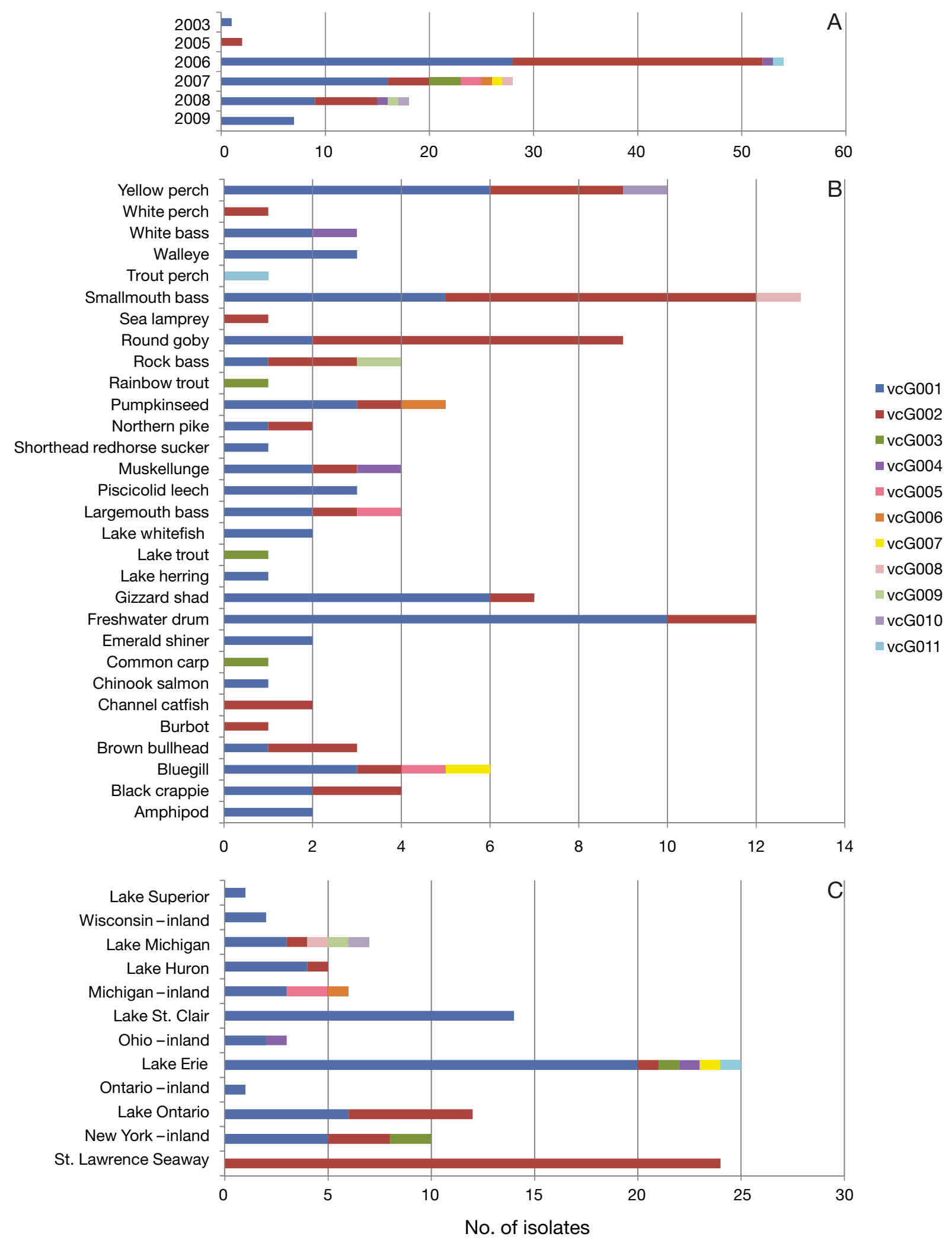

Fig. 4. Viral haemorrhagic septicemia virus. Distribution of 11 identified sequence types (vcG001-vcG011) among (A) different isolation years, (B) different host species, and (C) different geographic sub-regions defined within the Great Lakes study region 
another of the St. Lawrence Seaway, New York, and Lake Ontario, there is a clear pattern of predominance of vcG001 in the western and central sub-regions, with predominance of vcG002 in the eastern sub-regions. In total, sequence type vcG002 contained isolates from 6 of the 12 sub-regions, from every year between 2006 and 2008, and from 17 of the 30 host species.

Among 3 of the sequence types with a small number of isolates (vcG003, vcG004, and vcG005), some association was observed between sampling site and date, but to different degrees. Type vcG003 contained 3 isolates, 2 from different sites within New York in 2007 and 1 from Lake Erie, also in 2007. Type vcG004 had 2 isolates collected from Lake Erie in 2006 and from inland Ohio in 2008. Finally, both vcG005 isolates were from the same site in Budd Lake, inland Michigan, collected on the same date.

\section{Association with disease}

Although the information on disease associations of each virus isolate is not complete, it is known that 28 of the isolates were collected from asymptomatic fish, and 37 were from fish noted as either 'clinical', 'moribund', 'some mortality', or 'epidemic'. Among the 37 isolates from fish showing signs of disease, 23 were vcG001, 9 were vcG002, and 5 were in 1 of 3 other sequence types. Among the isolates from asymptomatic fish, 6 were vcG001 and 22 were vcG002. Of the 22 vcG002 isolates, 18 were collected from the St. Lawrence Seaway site within the same month as part of a healthy fish survey. Thus, the higher prevalence of vcG002 isolates among healthy fish may reflect the uneven sampling coverage, precluding the possibility of drawing conclusions about association of clinical disease with the different sequence types.

\section{Genetic diversity among virus isolates from same disease outbreak}

In 2 independent cases within the Great Lakes, genetic diversity was found within individual outbreaks of VHSV. In the first case, 3 isolates collected during a 2007 multi-species outbreak in Dunkirk Harbor on Lake Erie all showed different vcG sequences. Two isolates (TAVgr07-08 and TAVgr07-09, typed as vcG001 and vcG002, respectively) were isolated from gizzard shad, while the third isolate (TAVgr07-12), typed as vcG003, was from a common carp. In the second case, we typed 4 virus isolates from a large multispecies outbreak at Budd Lake in inland Michigan. The 4 isolates (TAVgr07-01, -02, -03, and -04), all collected in May 2007, were from bluegill, black crappie, largemouth bass, and pumpkinseed, respectively. The vcG sequences obtained from each of these isolates were vcG005, vcG001, a mixture of vcG001 abd vcG005, and vcG006, respectively.

\section{Genetic diversity within single VHSV isolates}

A related observation was the finding of genetic heterogeneity within 2 virus isolates, each of which had both the commonly observed sequence type vcG001 and a unique sequence type. These heterogeneous isolates (TAVgr07-03 and TAVgr07-14) were both from single fish, providing a useful demonstration that viruses with 2 different genetic types can co-infect a single fish in a natural setting. Isolate TAVgr07-14 was from a pumpkinseed collected in the Cayuga-Seneca Canal. This mixed infection was typed as vcG001 $(\sim 40 \%)$ and vcG002 ( 60\%). Even more interesting was the nature of the mixed infection in isolate TAVgr07-03, which was from a largemouth bass during an outbreak in Budd Lake, as described in the previous paragraph. Among the isolates from this outbreak, TAVgr07-03 was shown to have a mixed infection, typed as both vcG001 ( 45\%) and vcG005 $(\sim 55 \%)$, and 2 other isolates from the same outbreak were shown to have either vcG001 or vcG005.

\section{DISCUSSION AND CONCLUSIONS}

The emergence of VHSV in the Great Lakes region is a major veterinary event that - in order to limit the spread of disease-has led to a strong response in terms of regulation of aquaculture and baitfish industries. These include a USDA-APHIS federal order and amendments that restrict transport of fish from the Great Lakes region (USDA-APHIS 2008b), establishment of Ontario VHS baitfish management zones (Ontario MNR 2007), increased surveillance for virus in free-ranging fish by various state agencies and academic researchers (e.g. Bain et al. 2010), and active assessment of risk factors and measures to prevent further virus spread (VHSV Expert Panel \& Working Group 2010). The genetic typing of field isolates reported here adds to these efforts by providing insights into the genetic diversity, epidemiology, and possible origins of VHSV in the Great Lakes.

Compared with other fish rhabdovirus phylogenetic studies, the genetic diversity observed among VHSV isolates from the Great Lakes was extremely low. Out of 108 independent virus isolates, 61 were identical, with the sequence type vcG001 found in the index isolate MI03, and 47 differed from this sequence by only 1 nt. Thus, nearly all these isolates (98\%) differed from 
each other by no more than a single nucleotide in the region analyzed ( 1 of $669 \mathrm{nt}, 0.15 \%$ divergence). Only 2 isolates differed from vcG001 by $>1 \mathrm{nt}$. One of these was isolate TAVgr07-24 from Sturgeon Bay in Lake Michigan, which differed by 2 nt (vcG008), both of these encoding amino acid changes. The other was TAVgr08-03 from North Point Marina in Lake Michigan, which differed by $5 \mathrm{nt}$ (vcG009), leading to 3 amino acid changes. It is certainly likely that additional sequence differences exist within the $\mathrm{G}$ gene outside the sequencing window used here and elsewhere in the genomes of the various isolates, but it is unlikely that this region was uncharacteristically conserved in all these virus isolates. Additionally, parallel phylogenetic analyses using complete or partial N, G, and NV gene sequences of other sets of VHSV isolates have shown the same overall phylogenetic topography (Einer-Jensen et al. 2005). Thus, while future studies of longer genomic regions may enhance and refine our understanding of the genetic diversity among Great Lakes VHSV, these results indicate clearly that the isolates of VHSV currently circulating in the Great Lakes have extremely low diversity.

Between the 2 major sequence types identified here, vcG001 and vcG002, only a single nt difference was seen, and it did not encode an amino acid change. Thus, although there may be sequence differences elsewhere in the genome, it is certainly possible that no phenotypic difference exists between these viruses. This study was not designed to address the question of phenotypic differences. Instead, the value of the sequence differences identified is that they function as genetic tags that can be useful for molecular epidemiology of the viruses. Considering the connectivity within the Great Lakes watershed, it is most likely that the isolates with exactly identical sequences are epidemiologically linked, providing information on virus movement patterns. The alternative explanation, that the very same mutation may have occurred multiple times at random, while possible, is much less likely.

Within our data set, the pairwise genetic divergence ranged from 0.15 to $1.05 \%$ (from 1 to 7 nt difference out of $669 \mathrm{nt}$ ), and the great majority of isolates differed by only 0 to 2 nt. For comparison, Great Lakes VHSV vcG sequences differed from Nova Scotia and New Brunswick isolates by 2.1 to $2.7 \%$, and from North Pacific Ocean VHSV genotype IVa isolate sequences by 3.6 to $4.6 \%$. In phylogenies of full-length VHSV G gene sequences, the genetic diversity found within European genotypes I, II, and III was 1 to $6 \%$, $1 \%$, and 1 to $4 \%$, respectively, with the diversity among the 3 genotypes ranging from 9 to $13 \%$ (EinerJensen et al. 2004). In this 2004 report, the divergence between the European genotypes and the North Pacific Ocean genotype IVa isolates was from 13 to
$16 \%$. Thus, based on the overall global diversity known for VHSV, the low diversity among Great Lakes isolates supports the hypothesis that VHSV has only recently been introduced into the Great Lakes.

The 11 genetic types identified here expand our knowledge of the previously identified sub-lineage $\mathrm{IVb}$ of VHSV, and provide some interesting insights into molecular epidemiology when considered in light of the spatial geography of the isolation sites. For example, the finding of 3 different sequence types (vcG001, vcG002, and vcG003) among viruses from inland New York suggests introduction from diverse sources. The major inland waters of New York have waterway connections to Lakes Ontario and Erie as well as to the Hudson River via the New York State Barge Canal system. Given this fact, and the potential for anthropogenic movement of fish, inland New York isolates may have epidemiological links with either the Lake Ontario or Lake Erie watersheds, which harbor these sequence types. A second example is the finding of sequence type vcG004 in the Clear Fork Reservoir in inland Ohio and also in Lake Erie, which forms the northern border of Ohio. This is particularly interesting because the Clear Fork Reservoir is in the Mississippi River, not the Great Lakes, watershed, suggesting some epidemiological link other than water flow or natural fish movement.

Additional insights revealed by the typing involved heterogeneity of the virus types within single disease outbreaks. In Dunkirk Harbor on Lake Erie, the finding of 3 sequence types among only 3 virus isolates collected on the same day from the same fish kill clearly indicated genetic heterogeneity of the circulating virus. Similarly in Budd Lake, Michigan, the finding of 3 different sequence types among 4 isolates clearly indicated heterogeneity of the virus circulating during the disease outbreak. Similar heterogeneity has also been observed within a VHSV IVa epidemic in Pacific herring (K. A. Garver unpubl. data). These cases illustrate the value of analyzing multiple isolates from the same outbreak.

At a deeper level, our results also revealed 2 examples of genetic heterogeneity within virus isolates from single fish. The first isolate from an inland New York site had a mixture of the 2 most common sequence types, vcG001 and vcG002. The second was a fish from the 2007 Budd Lake VHS outbreak in Michigan, and it had a mixture of vcG001 and vcG005. This was particularly interesting because other isolates obtained from the same outbreak had either vcG001 or vcG005, so there was heterogeneity within the outbreak and within a single fish. Since sequence type vcG005 was not found in any other location, we can speculate that the virus introduced to Budd Lake was the dominant vcG001 type, and that sequence type vcG005 arose 
during viral replication in a fish in the lake. If this is true, isolate TAVgr07-03 may represent an active mutation event as it occurred de novo and became dominant within a single fish, or it could represent introduction of both sequence types into the same fish. Similar genetic heterogeneity has been reported previously for a small number of field isolates of European VHSV (Einer-Jensen et al. 2004), and for field isolates of the related fish rhabdovirus Infectious hematopoietic necrosis virus in Kamchatka, Russia (Rudakova et al. 2007), and in Europe (Enzmann et al. 2010).

The phylogenetic analysis presented here provided interesting insights into the relationship between Great Lakes IVb isolates and other VHSV isolates within major genogroup IV. As has been noted previously (Elsayed et al. 2006, Lumsden et al. 2007, Ammayappan \& Vakharia 2009), the Great Lakes IVb clade of VHSV isolates is clearly distinct from that of the North Pacific IVa clade. The position of the New Brunswick and Nova Scotia VHSV isolates close to the ancestral node for genogroup IV is consistent with the previous phylogeny (Gagné et al. 2007), and supports the hypothesis that the source of the VHSV that has emerged in the Great Lakes was most likely infected fish from the Atlantic coast of North America.

The extremely low genetic diversity among VHSV isolates from the Great Lakes region is consistent with recent introduction of a new virus into a watershed with abundant populations of naïve susceptible host species. This concept is supported by the lack of detection of VHSV prior to 2003, despite (1) ongoing health assessments of salmonid broodstock fish populations since the 1980s; (2) expanded wild fish broodstock surveys, including susceptible species such as muskellunge since 2001; and (3) periodic landscape-level surveys of wild fish conducted since 1996 as part of the National Wild Fish Health Survey (http://www.fws. gov/wildfishsurvey). These surveys all used isolation in cell culture to detect viruses. Additionally, fish kills of the scale experienced due to VHSV since 2005 had not been seen in the Great Lakes since the epidemics of bacterial kidney disease in the 1980s, and would not have gone unnoticed (G. Whelan, Michigan Department of Natural Resources, pers. comm.). The actual timing of introduction is uncertain, but it likely occurred several years prior to the first detection of the virus in 2003. We speculate that once the virus became established, its geographic range expanded rapidly, probably due to hydrologic connectivity and natural or anthropogenic movements of fish. Although the viral burden in the watershed has increased dramatically, no doubt aided by the virus's broad host range, genetic typing indicates that sequence divergence has not progressed rapidly. The genetic variation described in this report provides signs of early diversification, which may be facilitated by the broad geographic spread and stochastic factors of transmission in low-density freeranging host populations.

With regard to the origins of the sequence variation observed, the simplest hypothesis would be that VHSV with the most common sequence type, vcG001, was initially introduced into the Great Lakes, and the various sequence types observed have arisen within the watershed by mutation from this ancestor. In this scenario, the mutation to generate the vcG002 sequence type may have occurred relatively early in the emergence event, followed by localized spread and establishment throughout the watershed's eastern subregions. Alternatively, vcG002 may represent a second introduction event from a nearly identical source outside the Great Lakes. Considering the proximity of the St. Lawrence Seaway to the hypothesized Atlantic marine reservoir from which Great Lakes VHSV originated, it is also possible that a vcG002 isolate was the original type introduced, and mutation to vcG001 occurred early, spreading rapidly within the Great Lakes watershed. The single isolate with $5 \mathrm{nt}$ differences from vcG001 indicates that higher diversity does exist in the field, but it is likely to be relatively rare. In this case, we cannot distinguish between an unusually high level of mutation from a vcG001 ancestor and an independent introduction. Regardless, the high level of genetic identity among all VHSV isolates obtained from fish in the Great Lakes to date suggests that the disease outbreaks reported since 2005 comprise a single multi-year, multi-species epidemic due to a recently introduced virus.

In summary, this study provides a genetic analysis of VHSV isolates collected in the first $7 \mathrm{yr}$ (from 2003 to 2009) following the discovery and emergence of VHSV in the Great Lakes. This will provide a baseline for future comparison as the emergence event matures and the virus moves and potentially evolves within the new geographic range. This will be particularly important if Great Lakes VHSV gains access to aquaculture fish populations, or if it adapts to greater virulence in salmonid hosts, as occurred in European freshwater rainbow trout farms in the 1950s (Einer-Jensen et al. 2004). In experimental challenge studies, rainbow trout have been shown to be susceptible to infection but with relatively low mortality compared to other Great Lakes fish species (Kim \& Faisal 2010a,b). Continued monitoring will provide a unique opportunity to observe large-scale virus-host evolution in a natural aquatic system. Detailed information on the virus isolates characterized here is available in the Molecular Epidemiology of Aquatic Pathogens database, released in June 2010 (Emmenegger et al. in press, http://gis.nacse.org/vhsv). Fish health diagnosticians and researchers who isolate VHSV in North America 
are encouraged to provide virus to the authors for archiving and genetic typing, to facilitate future monitoring of the potential evolution of VHSV as it adapts to the Great Lakes ecosystem over time.

Acknowledgements. The authors thank all field researchers who collected fish tissue for analysis, laboratory staff who isolated the viruses, and the many participants who contributed their time and effort to providing background information associated with each virus isolate. We also thank Dr. K. EinerJensen for assistance with selection of appropriate representatives of European genotypes, and Dr. R. B. Life for assistance with the phylogenetic analyses. This work was supported by the Great Lakes Fisheries Trust (Grant \#08WRGR0006) and the US Department of Agriculture (USDA) Cooperative State Research, Education, and Extension Service (Award \#2007-38610-18387). Any use of trade names is for descriptive purposes only and does not imply endorsement by the US Government.

\section{LITERATURE CITED}

AFS-FHS (American Fisheries Society-Fish Health Section) (2007) FHS blue book: suggested procedures for the detection and identification of certain finfish and shellfish pathogens, American Fisheries Society-Fish Health Section, Bethesda, MD

Ammayappan A, Vakharia VN (2009) Molecular characterization of the Great Lakes viral hemorrhagic septicemia virus (VHSV) isolate from USA. Virol J 6:171-186

Bain MB, Cornwell ER, Hope KM, Eckerlin GE and others (2010) Distribution of an invasive aquatic pathogen (viral hemorrhagic septicemia virus) in the Great Lakes and its relationship to shipping. PLoS ONE 5:e10156

> Benmansour A, Basurco B, Monnier A, Vende P, Winton J, de Kinkelin P (1997) Sequence variation of the glycoprotein gene identifies three distinct lineages within field isolates of viral haemorrhagic septicaemia virus, a fish rhabdovirus. J Gen Virol 78:2837-2846

Einer-Jensen K, Ahrens P, Forsberg R, Lorenzen N (2004) Evolution of the fish rhabdovirus viral haemorrhagic septicaemia virus. J Gen Virol 85:1167-1179

Einer-Jensen K, Ahrens P, Lorenzen N (2005) Parallel phylogenetic analyses using the N, G or Nv gene from a fixed group of VHSV isolates reveal the same overall genetic typing. Dis Aquat Org 67:39-45

Elsayed E, Faisal M, Thomas M, Whelan G, Batts W, Winton J (2006) Isolation of viral haemorrhagic septicaemia virus from muskellunge, Esox masquinongy (Mitchill), in Lake St Clair, Michigan, USA reveals a new sublineage of the North American genotype. J Fish Dis 29:611-619

Emmenegger EJ, Kentop E, Thompson TM, Pittam S and others (in press) Development of an aquatic pathogen database (AquaPathogen X) and its utilization in tracking emerging fish virus pathogens in North America. J Fish Dis doi:10.111/j.1365-2761.2011.01270.x

Enzmann PJ, Castric J, Bovo G, Thiery R, Fichtner D, Schütze $\mathrm{H}$, Wahli $\mathrm{T}$ (2010) Evolution of infectious hematopoietic necrosis virus (IHNV), a fish rhabdovirus, in Europe over 20 years: implications for control. Dis Aquat Org 89:9-15

Faisal M, Schulz CA (2009) Detection of viral hemorrhagic septicemia virus (VHSV) from the leech Myzobdella lugubris Leidy, 1851. Parasit Vectors 2:45-48

Faisal M, Winters A (2011) Detection of viral hemorrhagic septicemia virus (VHSV) from Diporeia spp. (Pontoporei- idae, Amphipoda) in the Laurentian Great Lakes, USA. Parasit Vectors 4:2-5

Gagné N, MacKinnon AM, Boston L, Souter B, Cook-Versloot M, Griffiths S, Olivier G (2007) Isolation of viral haemorrhagic septicaemia virus from mummichog, stickleback, striped bass and brown trout in eastern Canada. J Fish Dis 30:213-223

Groocock GH, Getchell RG, Wooster GA, Britt KL and others (2007) Detection of viral hemorrhagic septicemia in round gobies in New York State (USA) waters of Lake Ontario and the St. Lawrence River. Dis Aquat Org 76:187-192

Hall BG (2008) Phylogenetic trees made easy: a how-to manual, 3rd edn. Sinauer Associates, Sunderland, MA

- Hedrick RP, Batts WN, Yun S, Traxler GS, Kaufman J, Winton JR (2003) Host and geographic range extensions of the North American strain of viral hemorrhagic septicemia virus. Dis Aquat Org 55:211-220

Kane-Sutton M, Kinter B, Dennis PM, Koonce JF (2010) Viral hemorrhagic septicemia virus infection in yellow perch, Perca flavescens, in Lake Erie. J Grt Lks Res 36:37-43

Kim R, Faisal M (2010a) Comparative susceptibility of representative Great Lakes fish species to the North American viral hemorrhagic septicemia virus Sublineage IVb. Dis Aquat Org 91:23-34

Kim R, Faisal M (2010b) Experimental studies confirm the wide host range of the Great Lakes viral haemorrhagic septicaemia genotype IVb. J Fish Dis 33:83-88

Kim SM, Lee JI, Hong MJ, Park HS, Park SI (2003) Genetic relationship of the VHSV (viral hemorrhagic septicemia virus) isolated from cultured olive flounder, Paralichthys olivaceus, in Korea. J Fish Pathol 16:1-12

> Lumsden JS, Morrison B, Yason C, Russell S and others (2007) Mortality event in freshwater drum Aplodinotus grunniens from Lake Ontario, Canada, associated with viral haemorrhagic septicemia virus, Type IV. Dis Aquat Org 76:99-111

Meyers TR, Winton JR (1995) Viral hemorrhagic septicemia virus in North America. Annu Rev Fish Dis 5:3-24

Ontario MNR (Ministry of Natural Resources) (2007) Ontario VHS baitfish management zones. http://www.mnr.gov.on. ca/en/Business/LetsFish/2ColumnSubPage/239471.html (Accessed November 2, 2010)

Nishizawa $T$, Iida $H$, Takano $R$, Isshiki $T$, Nakajima $K$, Muroga K (2002) Genetic relatedness among Japanese, American and European isolates of viral hemorrhagic septicemia virus (VHSV) based on partial G and P genes. Dis Aquat Org 48:143-148

OIE (Office International des Epizooties) (2009) Manual of diagnostic tests for aquatic animals. Office International des Epizooties, Paris

OIE (2010) Aquatic animal health code. Office International des Epizooties, Paris

Ronquist F, Huelsenbeck JP (2003) MRBAYES 3: Bayesian phylogenetic inference under mixed models. Bioinformatics 19:1572-1574

Rudakova SL, Kurath G, Bochkova EV (2007) Occurrence and genetic typing of infectious hematopoietic necrosis virus in Kamchatka, Russia. Dis Aquat Org 75:1-11

Schäperclaus W (1938) Die Schädigungen der deutschen Fischerei durch Fischparasiten und Fischkrankheiten. Allg Fischztg 41:256-259, 267-270

Skall HF, Olesen NJ, Mellergaard S (2005) Viral haemorrhagic septicaemia virus in marine fish and its implications for fish farming: a review. J Fish Dis 28:509-529

Smail DA (2000) Isolation and identification of Viral Haemorrhagic Septicaemia (VHS) viruses from cod Gadus morhua with the ulcus syndrome and from haddock Melanogram- 
mus aeglefinus having skin haemorrhages in the North Sea. Dis Aquat Org 41:231-235

Snow M, Bain N, Black J, Taupin V and others (2004) Genetic population structure of marine viral haemorrhagic septicaemia virus (VHSV). Dis Aquat Org 61:11-21

Tamura K, Dudley J, Nei M, Kumar S (2007) MEGA4: Molecular evolutionary genetics analysis (MEGA) software version 4.0. Mol Biol Evol 24:1596-1599

USDA-APHIS (US Department of Agriculture-Animal and Plant Health Inspection Service) (2008a) Regulated species susceptible to VHSV by the USDA. http://www.aphis. usda.gov/animal_health/animal_dis_spec/aquaculture/

Editorial responsibility: Julie Bebak, Auburn, Alabama, USA downloads/vhs_regulated_spp.pdf (Accessed November $20,2010)$

USDA-APHIS (2008b) Federal Order to prevent spread of VHSV. http://www.aphis.usda.gov/animal_health/animal_ dis_spec/aquaculture/downloads/vhs_fed_order_amended. pdf (Accessed November 20, 2010)

VHSV Expert Panel \& Working Group (2010) Viral hemorrhagic septicemia virus (VHSV IVb) risk factors and association measures derived by expert panel. Prev Vet Med 94:128-139

Wolf K (1988) Fish viruses and fish viral diseases. Cornell University Press, Ithaca, NY

Submitted: November 22, 2010; Accepted: March 23, 2011 Proofs received from author(s): July 19, 2011 